\title{
Photoacoustic effect measurement in aqueous suspensions of gold nanorods caused by low-frequency and low-power near-infrared pulsing laser irradiation
}

\author{
Cristina Sánchez López de Pablo, Julio Alberto Ramos Ávila, Tamara Fernández \\ Cabada, ${ }^{1}$ Francisco del Pozo Guerrero, and José Javier Serrano Olmedo
}

\begin{abstract}
When aqueous suspensions of gold nanorods are irradiated with a pulsing laser $(808 \mathrm{~nm})$, pressure waves appear even at low frequencies (pulse repetition rate of $25 \mathrm{kHz}$ ). We found that the pressure wave amplitude depends on the dynamics of the phenomenon. For fixed concentration and average laser current intensity, the amplitude of the pressure waves shows a trend of increasing with the pulse slope and the pulse maximum amplitude. We postulate that the detected ultrasonic pressure waves are a sort of shock waves that would be generated at the beginning of each pulse, because the pressure wave amplitude would be the result of the positive interference of all the individual shock waves. (C) 2013 Optical Society of America
\end{abstract}

\section{Introduction}

Gold nanorods (GNRs) have shown a huge potential for different biomedical applications because of their large light absorption and scattering cross sections in the near-infrared (NIR) region [1] (plasmon resonance). Thanks to this optical behavior, gold nanorods are suitable as sensors [2-6]. Moreover, GNRs are able to transform the absorbed energy into localized heat. This optical effect is used to develop cancer therapies [-11] such as photothermal tumor destruction either by direct increase of temperature or indirectly by coadjuvant drugs, either delivered by the particle or already present and activated by the heating.

Our research group has recently developed an optical hyperthermia device based on irradiation of GNRs with a cw laser in order to induce in vitro death of human brain astrocytoma cells (1321N1). The effectiveness of the method was determined by measuring changes in cell viability after laser irradiation of cells in the presence of GNRs. In accordance with other results in comparable experiments [12-14], ours indicated that continuous laser irradiation in the presence of the particles induced a 
significant decrease in cell viability, while no decrease in cell viability was observed with laser irradiation or incubation with GNRs alone [15].

Since the GNRs thermally expand when absorbing the light, pressure waves, also called photoacoustic waves, could be generated $[16,17]$. In this first work, we study the formation of photoacoustic waves when irradiating an aqueous suspension of GNRs with an NIR laser tuned for their surface plasmon resonance at low pulsing frequencies and low average power densities, as compared to other works [1-20].

\section{Material and Methods}

The cw laser (model H808, PSU-H-LED power source; Changchung New Industries, Changchun Jilin, China) works at $808 \mathrm{~nm}$, with a maximum output power of $5 \mathrm{~W}$, a beam height from the base of $29 \mathrm{~nm}$, a beam diameter at the aperture of 5 $8 \mathrm{~mm}$, and laser head dimensions of $155 \mathrm{~mm} \times$ $77 \mathrm{~mm} \times 60 \mathrm{~mm}$. The modulation of the laser was made using a pulse generator $(50 \mathrm{MHz}$ function/arbitrary/pulse generator TG5011 LXI; TTi, Huntingdon, UK), which was connected to the transistor-transistor logic (TTL) input of the laser. The TTL signal from the pulse generator allowed a pulsed laser signal $(0 \mathrm{~V}$, on; $5 \mathrm{~V}$, off) of different frequencies $(0-30 \mathrm{kHz})$ and duty cycles $(0 \%-100 \%)$ for different values of laser power/intensity. The shape of the laser pulses was measured using a large-area visible photoreceiver (model 2031; New Focus, Santa Clara, California). Table 1 shows the maximum values of the average current feeding the laser that the device is able to reach for different duty cycles.

The plasmon resonance (longitudinal band) of the used GNRs (30-10-808 Nanorodz; Nanopartz, Salt Lake City, Utah) is matched with the laser excitation wavelength at $808 \mathrm{~nm}$. The GNRs were dispersed in deionized water $(36 \mu \mathrm{g} / \mathrm{ml})$ with $0.1 \%$ ascorbic acid and $0.1 \%$ cetyltrimethylammonium bromide surfactant capping agent and had an axial diameter of $10 \mathrm{~nm}$ and a length of $41 \mathrm{~nm}$.

The laser was connected to the system via a multimode optical fiber with a core diameter of $600 \mu \mathrm{m}$, a length of $1.5 \mathrm{~m}$, and a power transmission of 90\%-99\% (600 mm multimode (MM) fiber; Changchung New Industries). The optical fiber was fixed vertically with the aid of a tripod stand and a burette clamp. The laser light from the fiber irradiated the samples through a collimating lens (78382; Newport, Irvine, California).

The ultrasonic measuring device is based on four piezoelectric receivers $(25 \mathrm{kHz}$ receiver; Kobitone Speakers \& Transducers, Mouser Electronics, Mansfield, Texas), around the irradiated samples. Their maximum sensitivity is located at $25 \mathrm{kHz}$.

Table 1. Maximum Average Current Feeding the Laser for Different Duty Cycles

\begin{tabular}{ccccccc}
\hline Duty cycle (\%) & 80 & 70 & 60 & 50 & 40 & 30 \\
\hline$I_{\text {MAX LASER }}(\mathrm{A})$ & 3.87 & 3.29 & 2.64 & 2.17 & 1.61 & 1.10 \\
\hline
\end{tabular}

The ultrasound receivers are selectable by the conditioning electronics to bring their signals to a lock-in amplifier (SR810 lock-in amplifier; Stanford Research Systems, Sunnyvale, California), whose reference input is the TTL signal that determines the frequency and duty cycle of the pulsing laser.

The experimental enclosure is a square insulated plastic vessel with the four ultrasonic sensors placed on each side of the square and fixed using commercial silicone. In addition, there is a polyurethane foam cover around the core of the plastic vessel, forming an isolated environment that is able to avoid bounces and echoes that may appear during the ultrasonic transmission. The polyurethane foam is indicated as acoustic and thermal insulation because it is solid, uniform, and durable. This material is able to absorb the acoustic waves and prevents wave overlaps and echoes that could result in measurement inaccuracies (Fig. 1).

A precision quartz cell with a light path of $10 \times 10 \mathrm{~mm}$ and dimensions of $46 \mathrm{~mm} \times 12.5 \mathrm{~mm} \times$ $12.5 \mathrm{~mm}$ (QS-111 SUPRASIL; Hellma GmbH \& Co. KG, Müllheim, Germany) is placed in the middle of the vessel to hold the aqueous suspensions of GNRs $(3 \mathrm{ml})$. The remaining hole is filled with deionized water in order to allow a more efficient transmission of the sound waves. The device is finally coated by a cover filled with foam that is in contact with the water surface in order to prevent any air layer that may hinder the acoustic signal reception.

During the experiments, the modulated laser light vertically irradiates the samples placed in the quartz cell. The laser pulse shape and magnitude are measured with a photoreceiver (large-area visible photoreceiver 2031; New Focus, Irvine, California). The frequency of the TTL modulation is $25 \mathrm{kHz}$, matching up with the maximum sensitivity frequency of the receivers. The sound waves are transmitted from the cell through the water to the ultrasonic sensors. The voltage signal output by the sensors is amplified by a factor of 100 and fed to the lock-in amplifier, which displays its value. As a whole we get a sensitivity of $-63 \mathrm{~dB}(0 \mathrm{~dB}=1 \mathrm{~V} / \mu \mathrm{bar})$. We can vary the average current intensity that feeds the laser, $I_{\text {LASER}}$, up to $5.0 \mathrm{~A}$ (the average laser power is linearly proportional to $I_{\text {LASER }}$ ) and the modulation duty cycle up to $80 \%$. We have used the duty cycle as an experimental parameter to change peak currents and slopes without changing the average laser power. The GNRs concentration is chosen as $0,36,72$, and $144 \mu \mathrm{g} / \mathrm{ml}$.

\section{Results and Discussion}

Figure 2 shows several laser pulse shapes for a fixed average laser current intensity $\left(I_{\mathrm{LASER}}=1.5 \mathrm{~A}\right)$ and the corresponding fitting curves for their rising edges. We used an exponential approach as follows: $y=A(1-\exp (-t / \tau)+B)$, where $A$ is the maximum amplitude of the exponential curve, $B$ is the small offset that the pulsed signals show, $\tau$ is the pulse rising time constant, $t$ is the time, and $y$ is the laser 


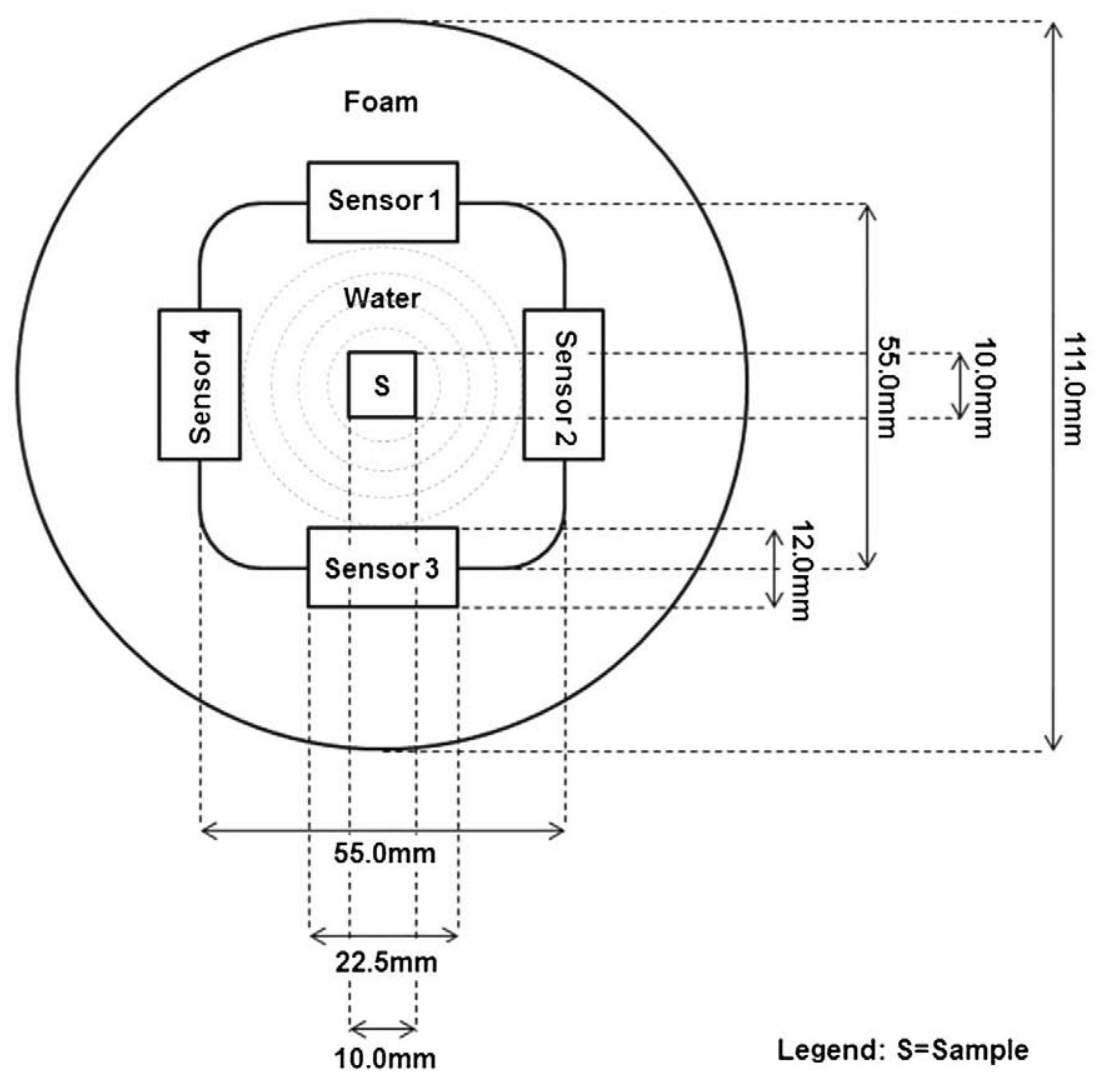

Fig. 1. Experimental enclosure diagram (view from above).

power. In order to keep constant the average laser current intensity, the signal peaks grow as duty cycles are shortened.

In Table 2 we summarize the main parameters from different pulsing regimes and the correspondence with their fitting curves. GNRs concentration $(36 \mu \mathrm{g} / \mathrm{ml})$ and average laser current intensity $\left(I_{\mathrm{LASER}}=1.50 \mathrm{~A}\right)$ were fixed. From the chosen fitting curve, the slope in $t=0 \mathrm{~s}, m$, and the maximum amplitude $A$ (absolute peak value less offset) are mathematically related as $m=y^{\prime}[t=0 \mathrm{~s}]=A / \tau$. As shown in Fig. 2 , the fit is quite good, revealing the rising of the laser power as a direct consequence of the dynamic of the power source that feeds the laser with current.

Figure 3 shows the correspondence between different duty cycles and maximum amplitude values $A$, obtained from the fit of the laser pulses. In order to determine if these results are consistent, we have calculated the theoretical correspondence between these parameters by taking into account the shape of our laser pulses. For this purpose, we have obtained and plotted the equation that links the parameters of interest through the calculation of the total amount of energy of each pulse. The shown good fit demonstrates that the ripples observed in

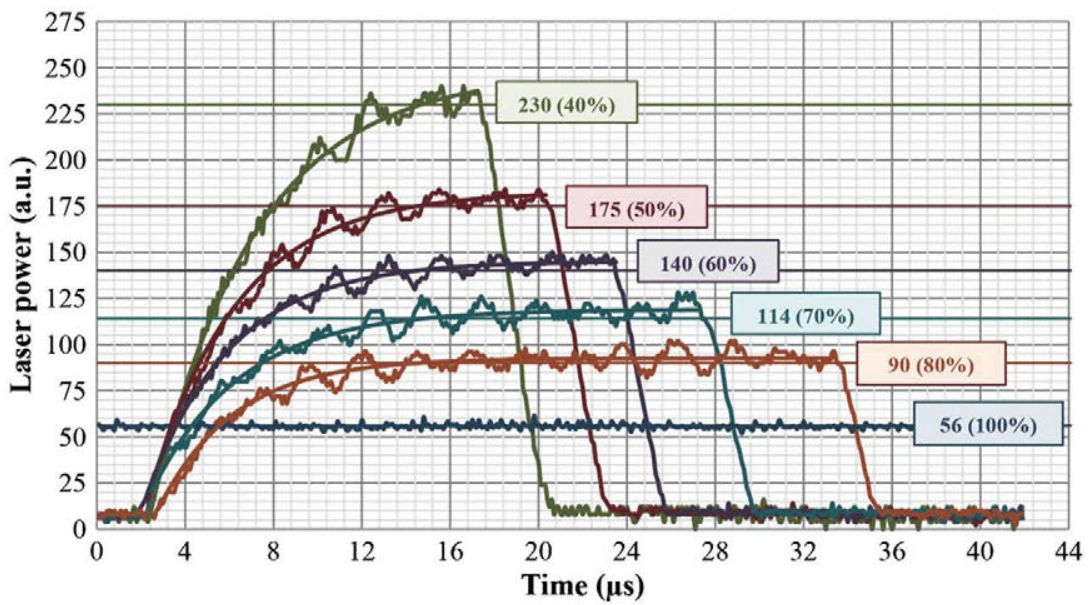

Fig. 2. Shape and peak values (labels) of laser power and fitting curves for different duty cycles (from $40 \%$ to $100 \%$ with $I_{\mathrm{LASER}}=1.5$ A). 
Table 2. Connection between the Parameters of the Laser Pulses and the Fitted Exponential Curves $\left(I_{\mathrm{LASER}}=1.50 \mathrm{~A}, 36 \mu \mathrm{g} / \mathrm{ml}\right)$

\begin{tabular}{lccccc}
\hline \multicolumn{2}{c}{ Laser Pulses } & & \multicolumn{2}{c}{ Fitted Curves } \\
\cline { 2 - 5 } Duty Cycle (\%) & Peak Power (a.u.) & & A Max. Amplitude (a.u.) & $\tau$ Time Constant (s) & $m$ Slope in $t=0 \mathrm{~s}$ \\
\hline 40.00 & 230.00 & 239.35 & 4.62 & 51.81 \\
50.00 & 175.00 & 171.29 & 3.99 & 32.93 \\
60.00 & 140.00 & 132.39 & 3.82 & 24.66 \\
70.00 & 114.00 & 100.35 & 4.08 & 3.43 \\
80.00 & 90.00 & 83.94 & 30 & 17.14 \\
\hline
\end{tabular}

Fig. $\underline{2}$ (an instrumental artifact) are not relevant for our results.

In Table 3 we show the voltage output by the lock-in amplifier for different GNRs concentrations, at a fixed duty cycle of $80 \%$ and average laser current intensity $\left(I_{\text {LASER }}=3.75 \mathrm{~A}\right)$.

Looking at Table $\underline{3}$ we can observe that the sensor 2 output is significantly lower than the others. This effect may be due to slight inaccuracies in the manual assembly of the device. Since this behavior is repeated whatever the measuring conditions, its outputs can be ignored.

Figure 4 shows that for a fixed value of the duty cycle $(80 \%)$ and for each average laser current intensity $\left(I_{\mathrm{LASER}}\right)$, the higher the concentration of the GNRs is, the greater the output voltage (average from the three correctly working sensors), so the higher the amplitude of the pressure signal. We can also see that there is a linear proportionality between the voltage and the GNR concentration. This result is consistent with the fact that the greater the GNR concentration is, the higher their efficiency to absorb the light $[21,22]$. Since the absorption phenomenon is independent for each particle [23], their thermal expansion is also independent. The waves produced by each one positively interfere, producing measurable pressure waves.

Figures $\underline{4}$ and $\underline{5}$ also show that the higher the average laser current intensity is, the greater the slope of the fitting lines and the pressure intensities (measured as sensor outputs). For samples without GNRs, the average voltage from the sensors is not zero but is proportional to the average laser current intensity, as we can see in Figs. 4 and 5 (represented as the offset value). This is a background effect due to the thermal behavior of the sample holder.

Figure $\underline{6}$ shows the sensor 3 output as a function of the duty cycle and of different average laser intensities, when using a fixed GNR concentration of $36 \mu \mathrm{g} / \mathrm{ml}$. We can observe that the voltage levels for each fixed laser current intensity and different duty cycles can be represented using a fitted line increasing for decreasing values of the duty cycle. Moreover, the higher the fixed average laser current intensity is, the higher pressure the signal that can be detected for a given duty cycle. This result shows that the observed phenomenon, the production of pressure waves at low frequencies, depends on both the average energy provided and the dynamics. This behavior is consistent with the fact that only under a so-called stress confinement condition [24], in which the duration of the laser pulse is less than a characteristic confinement time (a condition that is not satisfied in our case, because of the low frequency of the laser pulses), the amplitude of the acoustic wave launched by the optical absorbers depends only on the total amount of energy absorbed.

As we can observe in Table 2 , each value of the duty cycle corresponds to a maximum amplitude value. Moreover, this maximum amplitude is linearly

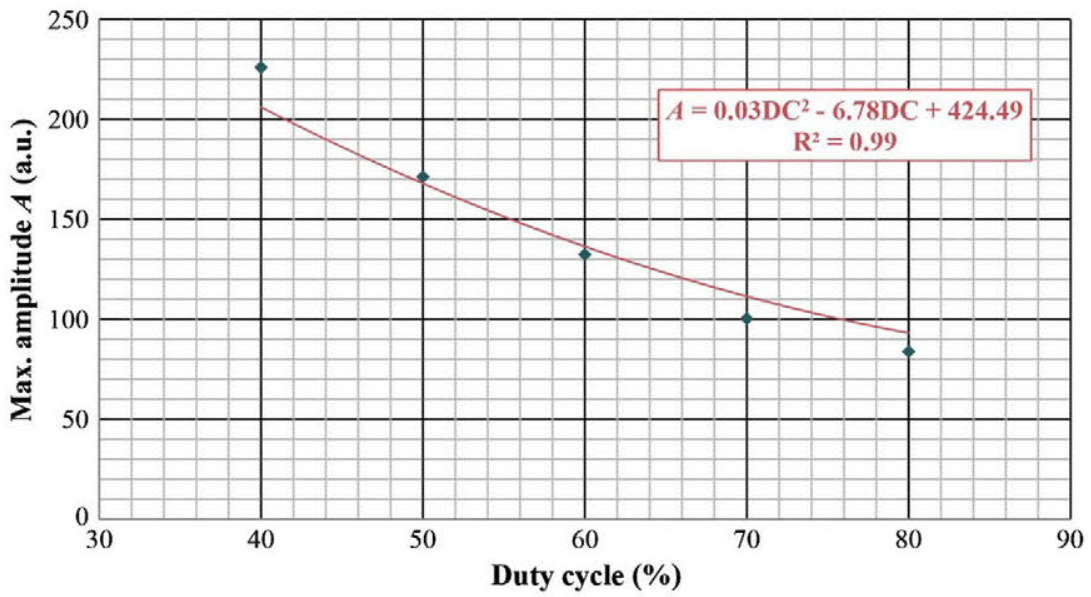

Fig. 3. Correspondence between different duty cycles and maximum amplitude values $A$ obtained from the fit of the laser pulses (diamonds), and fitted curve connecting these two parameters from the equations that define the total amount of energy of each laser pulse from an integration process to obtain the area under the pulses $(A=$ maximum amplitude (a.u.), DC $=$ duty cycle $(\%)$, mean squared error $=R^{2}, I_{\mathrm{LASER}}=1.50 \mathrm{~A}, 36 \mu \mathrm{g} / \mathrm{ml}$ ). 
Table 3. Lock-In Amplifier Output for Different Values of Gold Nanorod Concentration (Duty Cycle $\left.=80 \%, I_{\text {LASER }}=3.75 \mathrm{~A}\right)^{a}$

\begin{tabular}{lcccc}
\hline & \multicolumn{4}{c}{ Lock-In Output (nV) } \\
\cline { 2 - 5 } & Water & $36 \mu \mathrm{g} / \mathrm{ml}$ & $72 \mu \mathrm{g} / \mathrm{ml}$ & $144 \mu \mathrm{g} / \mathrm{ml}$ \\
\hline Sensor 1 & 94 & 192 & 321 & 562 \\
Sensor 2 & 75 & 148 & 248 & 512 \\
Sensor 3 & 98 & 232 & 315 & 546 \\
Sensor 4 & 104 & 253 & 345 & 561 \\
\hline
\end{tabular}

${ }^{a}$ Sensor 2 was not working well, so its outputs were discarded.

proportional to the slope in $t=0 \mathrm{~s}(\mathrm{~m})$, and the proportionality factor is given by the time constant $\tau$, as we can conclude from the definition of $m$. The parameter $\tau$ depends on the behavior of the instrument in the generation of laser pulses with different requirements of energy, duty cycle, or peak laser current intensity. In this case, the values of $\tau$ are very similar for all the generated laser pulses, so it can be taken as a constant.

Then, the dependence of the maximum amplitude value on the detected pressure waves is similar to its dependence on the slope in $t=0 \mathrm{~s}$, taking into account the proportionality factor $(\tau)$. Note that this effect is characteristic of our system and can not necessarily be extended to other systems, because the shape of the laser pulses and the subsequent fitted curves depend on the power supply.

Once the dependence of the data on the duty cycle is eliminated, in Figs. 7 and 8 we present the dependence of the detected voltage levels on the maximum amplitude $A$ and the slope at the beginning of the rising edges of the pulses. The crossing at zero of the fitted lines has been forced. Actually, when laser irradiation is not pulsed, the maximum amplitude value $A$ and the slope in $t=0 \mathrm{~s}$ should be zero because there are no pressure waves generation, and

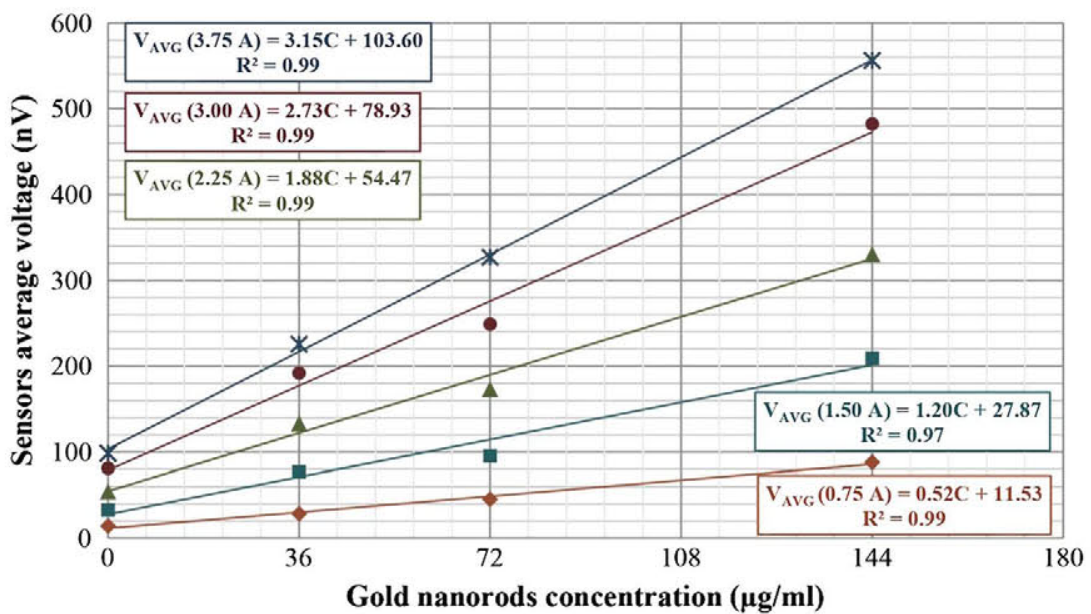

Fig. 4. Average voltage from sensors 1,3 , and 4, and fitting lines for different concentrations. Duty cycle $=80 \%$ and $I_{\mathrm{LASER}}$ in the range $0.75-3.75 \mathrm{~A}(0.75 \mathrm{~A}$, diamonds; $1.50 \mathrm{~A}$, squares; $2.25 \mathrm{~A}$, triangles; $3.00 \mathrm{~A}$, circles; and $3.75 \mathrm{~A}$, asterisks), with steps of $0.75 \mathrm{~A}$ [sensor average voltage $(\mathrm{nV})=V_{\mathrm{AVG}}, \mathrm{GNR}$ concentration $(\mu \mathrm{g} / \mathrm{ml})=C$, mean squared error $\left.=R^{2}\right]$.

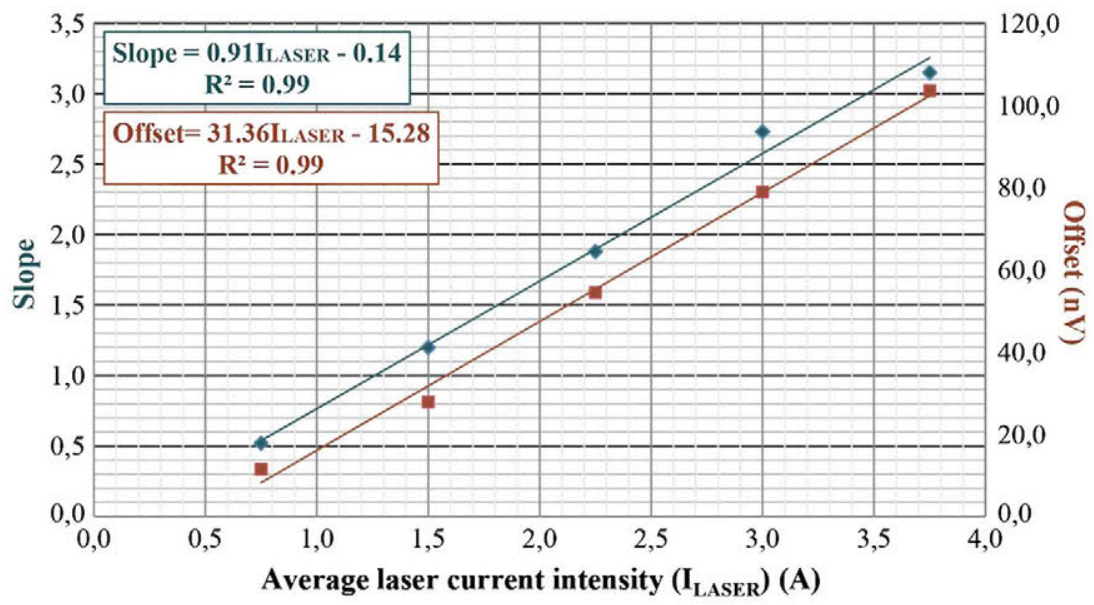

Fig. 5. Slopes (diamonds) and offset values (squares) of the fitted lines obtained in Fig. $\underline{4}$ for different values of average laser intensities $\left(I_{\text {LASER }}\right)$ and a fixed duty cycle of $80 \%$ (mean squared error $=R^{2}$ ). 


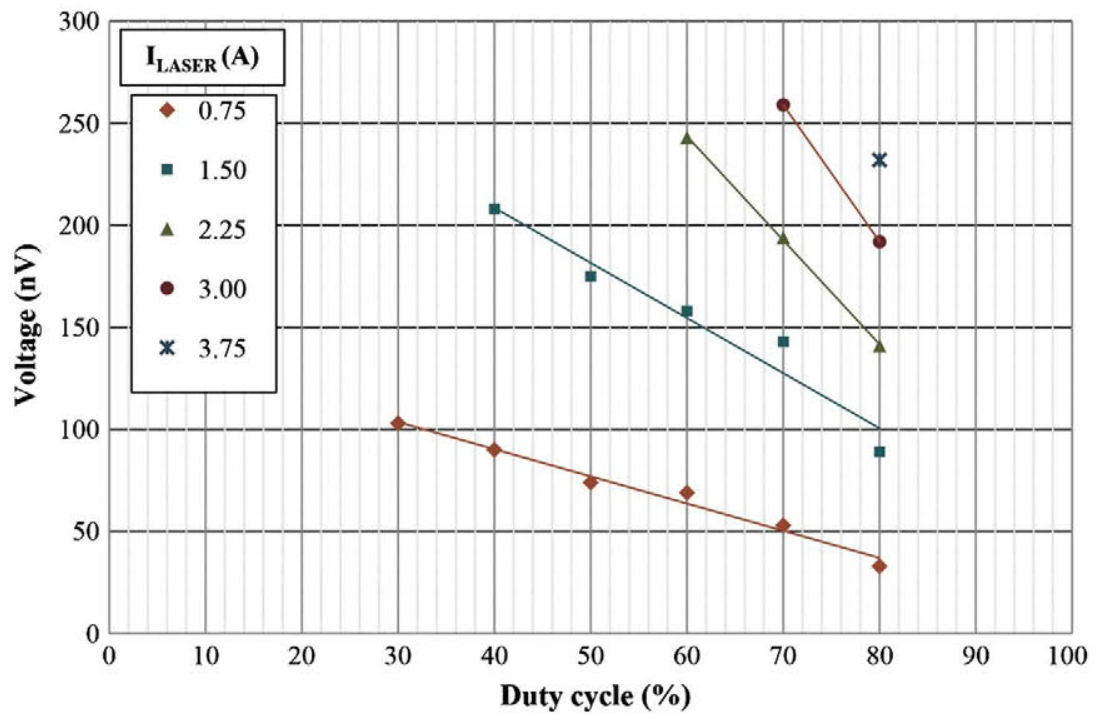

Fig. 6. Sensor 3 output and fitting lines for different laser intensities in a duty cycle sweep (GNR concentration of $36 \mu \mathrm{g} / \mathrm{ml}$ ).

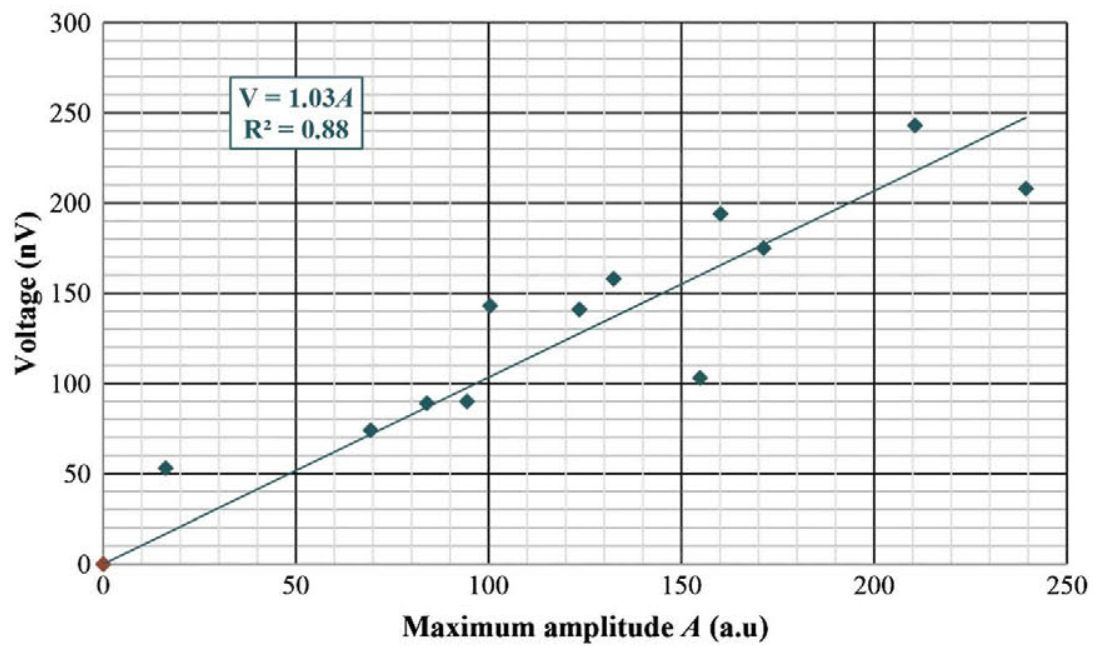

Fig. 7. Dependence of the voltage levels on the maximum amplitude value $A$ of the rising edges of the pulses [voltage $(\mathrm{nV})=V$, maximum amplitude value (a.u.) $=A$, mean squared error $=R^{2}$ ] for different average laser current intensities $\left(I_{\mathrm{LASER}}=0.75\right.$, 1.50 , and $2.25 \mathrm{~A})$ in a duty cycle sweep $(36 \mu \mathrm{g} / \mathrm{ml}$, sensor 3$)$.

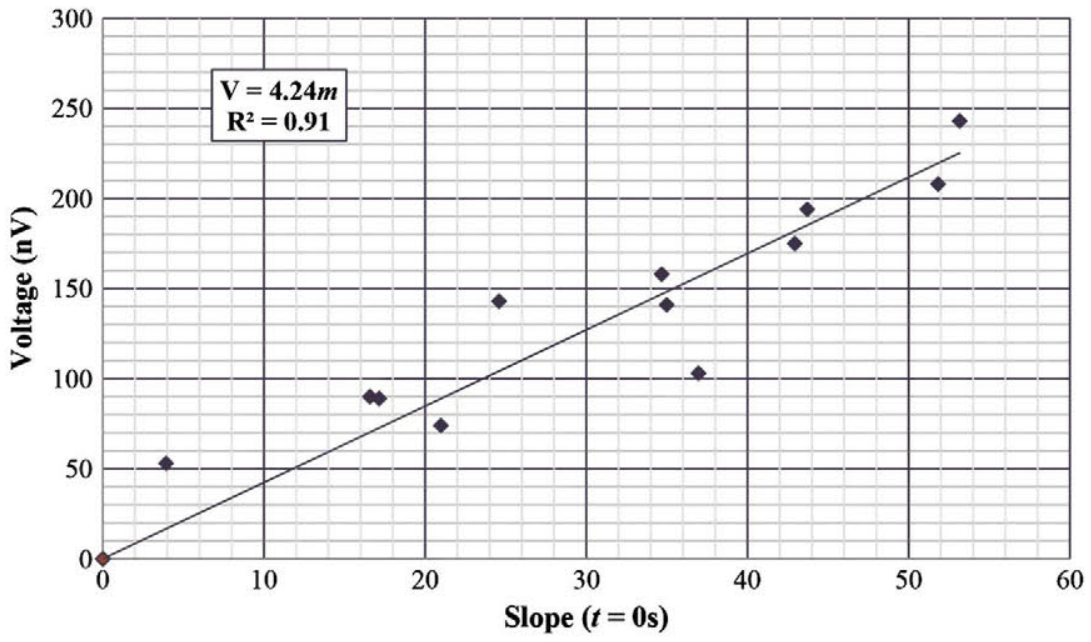

Fig. 8. Dependence of the voltage levels on the slope $(t=0 \mathrm{~s})$ of the rising edges of the pulses [voltage $(\mathrm{nV})=V$, slope $(t=0 \mathrm{~s})=m$, mean squared error $=R^{2}$ ] for different average laser current intensities $\left(I_{\mathrm{LASER}}=0.75,1.50\right.$, and $\left.2.25 \mathrm{~A}\right)$ in a duty cycle sweep (36 $\mu \mathrm{g} / \mathrm{ml}$, sensor 3). 
the voltage detected by the lock-in amplifier should also be zero.

If the pulses were ideal, the slopes in $t=0 \mathrm{~s}$ would approach infinity. However, in our real case the pulses are not ideal, and the slopes change depending on the duty cycle. Both the slope in $t=0 \mathrm{~s}$ and the $A$ values exhibit a trend of increasing with respect to the detected voltage level, and hence with the wave pressure. As expected, they are similar for different average laser current intensities. The result shown is consistent with previous theoretical studies of photoacoustic propagation [24,25], in which it is stated that in the limit of highly localized, almost instantaneous optical absorption, as with gold nanorods, the acoustic pulse shape is approximated by the time derivative of the optical pulse. Nevertheless, according to the results and taking into account the behavior of our instrument, we cannot determine whether the actual linear dependence of the pressure waves is associated with the derivative of the laser pulse or with the maximum amplitude.

Finally, as the sensitivity of the ultrasonic receivers is $-63 \mathrm{~dB}(0 \mathrm{~dB}=1 \mathrm{~V} / \mu \mathrm{bar})$, the conversion from acoustic pressure to output voltage is expressed as $10(-63 \mathrm{~dB} / 20)=7.08 \cdot 10^{-4} \mathrm{~V} / \mu \mathrm{bar}$. The voltage levels obtained from the lock-in amplifier in this work were in the range $30-600 \mathrm{nV}$, so taking into account that the signal conditioning circuit has an electrical gain of 100, the amplitude of the pressure signals detected by the ultrasonic receivers $\left(P_{\text {sensor }}\right)$ is in the range $0.04-0.85 \mu \mathrm{Pa}(1 \mathrm{bar}=105 \mathrm{~Pa})$. The distance from the quartz cell to each sensor is $16.5 \mathrm{~mm}(r)$, and the distance from the center of the sample to one side of the quartz cell is $5.0 \mathrm{~mm}$ $\left(r_{\text {cell }}\right)$, as can be obtained from Fig. 1. Since the sound wave starts in the quartz cell and travels through the water environment toward the sensors, the pressure amplitude at the border of the cell $\left(P_{0}\right)$ can be estimated, taking into account the ratio between the squared distances between sensors and the cell size, to yield $P_{0}=10.89 \cdot P_{\text {sensor }}$.

\section{Conclusion}

We have developed a device to measure and characterize the photoacoustic effect that appears when an aqueous suspension of GNRs is irradiated with a lowfrequency and low-power NIR pulsing laser. We have found that the magnitude of the wave pressure depends not on the total amount of provided energy but on the dynamics of the phenomenon. The pressure magnitude shows a trend of increasing, probably linearly, with respect to the slope in $t=0 \mathrm{~s}$ of the pulses, and hence with respect to the maximum amplitude value. In our system, the slope and the maximum amplitude are linearly proportional. However, we postulate that the measured phenomenon of pressure wave generation is the result of the coexistence of many processes taking place at each individual nanorod. As they thermally expand very quickly at the beginning of each laser pulse, the addition of all the individual shock waves generated by each one would positively interfere to form the observed transient phenomenon. This effect would take place despite of the smooth rising edges in our setup as compared to other excitation systems.

In short, we can say that the thermal expansion of gold nanorods suspended in water under a lowfrequency exciting laser light generates acoustic waves that are quantifiable as proposed. The magnitude of these signals can be controlled through the modification of the laser and modulation parameters. The determination of the real influence of these effects in biomedical applications and the correct theoretical explanation are important tasks for the future research.

\section{References}

1. W. Ni, X. Kou, Z. Yang, and J. Wang, "Tailoring longitudinal surface plasmon wavelengths, scattering, and absorption cross sections of gold nanorods," ACS Nano 2, 677-686 (2008).

2. C. Yu and J. Irudayaraj, "Multiplex biosensor using gold nanorods," Anal. Chem. 79, 572-579 (2007).

3. H. Huang, X. Liu, Y. Zeng, X. Yu, B. Liao, P. Yi, and P. K. Chu, "Optical and biological sensing capabilities of $\mathrm{Au}_{2} \mathrm{~S} / \mathrm{AuAgS}$ coated gold nanorods," Biomaterials 30, 5622-5630 (2009).

4. G. K. Darbha, U. S. Rai, A. K. Singh, and P. C. Ray, "Goldnanorod-based sensing of sequence specific HIV-1 virus DNA by using hyper-Rayleigh scattering spectroscopy," Chem. Eur. J. 14, 3896-3903 (2008).

5. L. Tong, Q. Wei, A. Wei, and J. X. Cheng, "Gold nanorods as contrast agents for biological imaging: optical properties, surface conjugation and photothermal effects," Photochem. Photobiol. 85, 21-32 (2009).

6. S. Ha, A. Carson, A. Agarwal, N. A. Kotov, and K. Kim, "Detection and monitoring of the multiple inflammatory responses by photoacoustic molecular imaging using selectively targeted gold nanorods," Opt. Express 2, 645-657 (2011).

7. G. Von Maltzahn, J. H. Park, A. Agrawal, N. K. Bandaru, S. K. Das, M. J. Sailor, and S. N. Bhatia, "Computationally guided photothermal tumor therapy using long circulating gold nanorod antennas," Cancer Res. 69, 3892-3900 (2009).

8. C. A. Peng and C. H. Wang, "Anti-neuroblastoma activity of gold nanorods bound with GD2 monoclonal antibody under near-infrared laser irradiation," Cancers 3, 227-240 (2011).

9. G. S. Terentyuk, A. V. Ivanov, N. I. Polyanskaya, I. L. Maksimova, A. A. Skaptsov, D. S. Chumakov, B. N. Khlebtsov, and N. G. Khlebtsov, "Photothermal effects induced by laser heating of gold nanorods in suspensions and inoculated tumours during in vivo experiments," Quantum Electron 42, 380-389 (2012).

10. A. M. Alkilany, L. B. Thompson, S. P. Boulos, P. N. Sisco, and C. J. Murphy, "Gold nanorods: their potential for photothermal therapeutics and drug delivery, tempered by the complexity of their biological interactions," Adv. Drug Delivery Rev. 64, 190-199 (2012).

11. R. Jaskula-Sztul, Y. Xiao, A. Javadi, J. Eide, W. Xu, M. Kunnimalaiyaan, S. Gong, and H. Chen, "Multifunctional gold nanorods for targeted drug delivery to carcinoids," J. Surg. Res. 172, 235 (2012).

12. L. Tong, Y. Zhao, T. B. Huff, M. N. Hansen, A. Wei, and J. X. Cheng, "Gold nanorods mediate tumor cell death by compromising membrane integrity," Adv. Mater. 19, 3136-3141 (2007).

13. E. C. Dreaden, M. A. Mackey, X. Huang, B. Kang, and M. A. El-Sayed, "Beating cancer in multiple ways using nanogold," Chem. Soc. Rev. 40, 3391-3404 (2011).

14. W. I. Choi, A. Sahu, Y. H. Kim, and G. Tae, "Photothermal cancer therapy and imaging based on gold nanorods," Ann. Biomed. Eng. 40, 534-546 (2012) 
15. T. Fernández, C. Sánchez, A. Martínez, F. del Pozo, J. J. Serrano, and M. Ramos, "Induction of cell death in a glioblastoma line by hyperthermic therapy based on gold nanorods," Int. J. Nanomedicine 7, 1511-1523 (2012).

16. V. Pustovalov and V. Zharov, "Threshold parameters of the mechanisms of selective nanophotothermolysis with gold nanoparticles," Proc. SPIE 6854, 685412 (2008).

17. M. Hu, X. Wang, G. V. Hartland, P. Mulvaney, J. P. Juste, and J. E. Sader, "Vibrational response of nanorods to ultrafast laser induced heating: theoretical and experimental analysis," J. Am. Chem. Soc. 125, 14925-14933 (2003).

18. R. R. Letfullin, C. Joenathan, T. F. George, and V. P. Zharov, "Laser-induced explosion of gold nanoparticles: potential role for nanophotothermolysis of cancer," Nanomedicine $\mathbf{1}$, 473-480 (2006).

19. R. R. Letfullin, T. F. George, G. C. Duree, and B. M. Bollinger, "Ultrashort laser pulse heating of nanoparticles: comparison of theoretical approaches," Adv. Opt. Technol. 2008, 251718 (2008).
20. J. Shah, S. Park, S. Aglyamov, T. Larson, L. Ma, K. Sokolov, K. Johnston, T. Milner, and S. Y. Emelianov, "Photoacoustic imaging and temperature measurement for photothermal cancer therapy," J. Biomed. Opt. 13, 034024 (2008).

21. J. R. Cole, N. A. Mirin, M. W. Knight, G. P. Goodrich, and N. J. Halas, "Photothermal efficiencies of nanoshells and nanorods for clinical therapeutic applications," J. Phys. Chem. B 113, 12090-12094 (2009).

22. V. P. Pattani and J. W. Tunnell, "Nanoparticle-mediated photothermal therapy: a comparative study of heating for different particle types," Lasers Surg. Med. 44, 675-684 (2012).

23. L. Novotny and B. Hecht, Principles of Nano-Optics (Cambridge University, 2006)

24. S. Y. Emelianov, P. C. Li, and M. O'Donnell, "Photoacoustics for molecular imaging and therapy," Phys. Today 62(8), $34-39$ (2009).

25. G. J. Diebold and T. Sun, "Properties of photoacoustic waves in one, two, and three dimensions," Acta Acust. United Acust. 80, 339-351 (1994). 